\title{
DECENTRALIZED CONTROL OF LARGE-SCALE UNCERTAIN NONLINEAR SYSTEMS BY LINEAR OUTPUT FEEDBACK*
}

\author{
MICHAEL T. FRYE ${ }^{\dagger}$, CHUNJIANG QIAN $^{\dagger}$, AND RICHARD COLGREN ${ }^{\ddagger}$
}

\begin{abstract}
This paper studies the problem of global decentralized control by output feedback for large-scale uncertain systems whose subsystems are interconnected not only by their outputs but also by their unmeasurable states. We show that under a linear growth condition, there is a decentralized output feedback controller rendering the closed-loop system globally exponentially stable. This is accomplished by extending an output feedback domination design that requires only limited information about the nonlinear system. We will apply our design to lower, upper, and non-triangular nonlinear systems. The significance of our results is that we do not need to have prior information about the nonlinearities of the system. Furthermore, we need to only employ a linear observer in combination with a linear controller to stabilize the system. A time-varying output feedback controller is also constructed for use with large-scale systems that have unknown parameters.
\end{abstract}

1. Introduction. Decentralized control of interconnected systems has been an area of considerable research due to its obvious practical application to current problems in the field of controls. Large-scale systems have very complex dynamic models due to the uncertain environment, the varying system parameters, and the interconnected structure of the system. Also it is inevitable that nonlinearities are prevalent throughout the dynamics of the interconnected systems. All these issues make the stabilization of such large-scale systems a difficult control problem. Though quite challenging, the research of large-scale systems are relevant to such areas as communication networks, satellite constellations, and the formation control of autonomous vehicles.

The research of large-scale nonlinear systems began in the late 1960's and early 1970 's. One of the earliest investigations into the nonlinear issues of large-scale systems centered around time-varying stabilization [2]. The early research in [7] demonstrated a method of using high-gain state feedback to stabilize the nonlinearities of the large-scale systems. The research in the early 1980's focused on the use of state feedback to globally stabilize large-scale nonlinear systems. Adaptive control was applied in [3] to stabilize a class of large-scale nonlinear systems with success. Output feedback had also been applied to linear large-scale system in such papers as [1], [15],

${ }^{*}$ This material is based upon work partially supported by the National Science Foundation under Grant No. ECS-0239105 (UTSA), EPS-0236913 (KU), and matching support from the University of Kansas.

${ }^{\dagger}$ Department of Electrical and Computer Engineering, The University of Texas at San Antonio, San Antonio, Texas 78249, E-mail: Michael.Frye@utsa.edu, Chunjiang.Qian@utsa.edu

${ }^{\ddagger}$ Department of Aerospace Engineering, The University of Kansas, Lawrence, Kansas 66045, Email: rcolgren@ku.edu 
and [11] during the same time. The use of output feedback has certain apparent advantages because of the fact that not all of the state variables of a large-scale system can be measured. An interesting paper [18], which applied static output feedback to nonlinear large-scale systems, used a linear quadratic method to develop a sufficient condition for stability of the large-scale system on a closed connected set. The use of adaptive control and output feedback was applied in [5] and [4]. [6] discussed output feedback and disturbance rejection for large-scale systems with disturbances while the issue of robustness of output feedback was studied in [10].

Most of the existing decentralized output feedback results are developed for largescale systems interconnected only by the outputs. There are very few results dealing with large-scale systems interconnected by unmeasurable states. One existing result is the work [18] which dealt with nonlinear functions that can depend on unbounded unmeasurable states, however the result is not a global one. In fact, due to the use of a quadratic method, the static output controller proposed in [18] could only stabilize the system on a closed connected set. Currently, the problem of global decentralized control of large-scale systems interconnected by unbounded unmeasurable states is quite open. The major difficulty in implementing an output feedback controller for highly interconnected large-scale systems is due to the fact that for each subsystem, the presence of unmeasurable states of the other subsystems makes the design of the decentralized output feedback controller very complicated. In other words, it is very challenging to design a global stabilizer for one subsystem only using its output while this subsystem is also driven by the unmeasurable states of the other subsystems.

When the nonlinear functions are not Lipschitz in unmeasurable states or have uncertainties associated with unmeasurable states, the paper [13] was able to provide a method of constructing a linear output feedback stabilizer using a feedback domination design method under a linear growth condition. In this paper, we extend the results from [13] and apply them to $m$ subsystems that are highly interconnected through all the unmeasurable states. Under a linear growth condition imposed on the uncertain nonlinear vector fields, we will design a linear controller for each subsystem using only its own output. As shown in [13], this output feedback controller needs no information of the uncertain nonlinearities. The new structure of the observer and controller will enable us to overcome the difficulty in dealing with the output feedback control problem in the presence of unmeasurable states in each subsystem. A combination of the observers and controllers constructed for each subsystem will globally stabilize the whole large-scale system. Our design method will be implemented for lower-triangular, upper-triangular, and non-triangular uncertain nonlinear systems.

This paper is organized as follows: Section 2 introduces the Problem Statement, where we will present our assumption that utilizes a growth condition for bounding the nonlinearities of $m$ subsystems. In Section 3, we will present our main result for lower-triangular systems and demonstrate that a linear observer coupled with its 
output feedback controller can globally stabilize a large-scale system comprised of $m$ subsystems. Section 4 extends our design method to upper-triangular nonlinear systems. In Section 5, we extend the results to systems with non-triangular structures and when the growth rate is unknown. Finally we summary our results in Section 6 .

2. Problem Statement. In this paper, we consider the following class of largescale uncertain nonlinear systems comprised of $m$ subsystems,

$$
\begin{gathered}
\text { Subsystem 1: }\left\{\begin{array}{c}
\dot{x}_{11}=x_{12}+f_{11}(x, d(t)) \\
\dot{x}_{12}=x_{13}+f_{12}(x, d(t)) \\
\vdots \\
\dot{x}_{1 n}=u_{1}+f_{1 n}(x, d(t)) \\
y_{1}=x_{11}
\end{array}\right. \\
\vdots \\
\text { Subsystem i: }\left\{\begin{array}{c}
\dot{x}_{i 1}=x_{i 2}+f_{i 1}(x, d(t)) \\
\dot{x}_{i 2}=x_{i 3}+f_{i 2}(x, d(t)) \\
\vdots \\
\dot{x}_{i n}=u_{i}+f_{i n}(x, d(t)) \\
y_{i}=x_{i 1} \\
\vdots \\
\dot{x}_{m 1}=x_{m 2}+f_{m 1}(x, d(t)) \\
\dot{x}_{m 2}=x_{m 3}+f_{m 2}(x, d(t)) \\
\vdots \\
\dot{x}_{m n}=u_{m}+f_{m n}(x, d(t)) \\
y_{m}=x_{m 1}
\end{array}\right.
\end{gathered}
$$

where $x_{i}=\left(x_{i 1}, \cdots, x_{i n}\right), i=1, \cdots, m, x=\left(x_{1}, \cdots, x_{m}\right)$, is the state, $y_{i}$ is the output, $u_{i}$ is the control input, $d(t)$ is a bounded unknown disturbance, and $f_{i j}$ is a function satisfying the following condition.

Assumption 2.1. For $i=1, \cdots, m$ and $j=1, \cdots, n$, there is a constant $c \geq 0$ such that

$$
\begin{aligned}
\left|f_{i j}(x, d(t))\right| \leq & c\left(\left|x_{11}\right|+\cdots+\left|x_{1 j}\right|+\left|x_{21}\right|+\cdots\right. \\
& \left.+\left|x_{2 j}\right|+\cdots+\left|x_{m 1}\right|+\cdots+\left|x_{m j}\right|\right) .
\end{aligned}
$$

The objective of this paper is to design a dynamic compensator and controller of the form

$$
\begin{aligned}
& \dot{\xi}_{i}=M \xi_{i}+N y_{i}, \quad M \in \mathbb{R}^{n \times n}, \quad N \in \mathbb{R}^{n} \\
& u_{i}=K \xi_{i}, \quad K \in \mathbb{R}^{1 \times n}, i=1, \cdots, m
\end{aligned}
$$


such that the closed-loop system (2.1) and (2.3) is globally exponentially stable (GES) at the equilibrium $(x, \xi)=(0,0)$.

Remark 2.2. System (2.1) under Assumption 2.1 represents a class of largescale systems whose subsystems are interconnected not only by their outputs but also by their unmeasurable states. Moreover, those unmeasurable states in (2.1) will not disappear in the bounding functions in (2.2). For example, in the following system

$$
\begin{aligned}
& \text { Subsystem } 1\left\{\begin{array}{l}
\dot{x}_{11}=x_{12} \\
\dot{x}_{12}=u_{1}+d(t) x_{12}+d(t) x_{22}, \\
y_{1}=x_{11}, \\
\text { where } \quad d(t) \in[1,2]
\end{array}\right. \\
& \text { Subsystem 2 }\left\{\begin{array}{l}
\dot{x}_{21}=x_{22} \\
\dot{x}_{22}=u_{2}+\ln \left(1+x_{12}^{2}\right)+x_{22} \sin \left(x_{22}\right) \\
y_{2}=x_{21} .
\end{array}\right.
\end{aligned}
$$

Subsystem 1 and Subsystem 2 are interconnected through the unmeasurable state $x_{12}$ and $x_{22}$ that cannot be eliminated even in the bounding functions. Due to this reason, the problem of global decentralized control of Subsystem 1 and Subsystem 2 by output feedback is challenging.

This paper will show how a linear output feedback controller of the form (2.3) can be recursively constructed to globally stabilize system (2.1) under Assumption 2.1. An advantage of our design method is that the precise knowledge of the nonlinearities or uncertainties of the systems does not need to be known. What is really needed is the growth rate $c$ of the bounding function of the uncertain nonlinearities as shown in Assumption 2.1. This feature makes it possible to stabilize $m$ uncertain interconnected subsystems using very limited information even though the subsystems are interconnected through unmeasurable states.

3. Output Feedback Domination with High Gain. In this section, we prove that under Assumption 2.1 there exists a globally stabilizing output feedback controller for system (2.1). This is done by using a new output feedback domination design which explicitly constructs a linear output feedback control law without requiring the precise knowledge of the nonlinearities in system (2.1).

TheOREm 3.1. Under Assumption 2.1, there exists a linear output feedback controller (2.3) that renders the large-scale interconnected system (2.1) globally exponentially stable.

Proof. In order to prove Theorem 3.1, we utilize the output feedback domination design first proposed in [13] to design a linear observer and controller for each individual subsystem. With these $m$ observers and controllers, it can be shown that the closed-loop system is globally exponentially stable after a large enough gain has been carefully chosen. 


\section{A. LINEAR OBSERVER DESIGN}

We begin by designing the following linear observer for subsystem $i$

$$
\begin{aligned}
\dot{\hat{x}}_{i 1}= & \hat{x}_{i 2}+L a_{1}\left(x_{i 1}-\hat{x}_{i 1}\right) \\
& \vdots \\
\dot{\hat{x}}_{i(n-1)}= & \hat{x}_{i n}+L^{(n-1)} a_{n-1}\left(x_{i 1}-\hat{x}_{i 1}\right) \\
\dot{\hat{x}}_{i n}= & u_{i}+L^{n} a_{n}\left(x_{i 1}-\hat{x}_{i 1}\right)
\end{aligned}
$$

where $L \geq 1$ is a gain parameter to be determined later, and $a_{j}>0, j=1, \cdots, n$, are coefficients of the Hurwitz polynomial $p(s)=s^{n}+a_{1} s^{(n-1)}+\cdots+a_{n-1} s+a_{n}$. Define the following error term $e_{i j}=x_{i j}-\hat{x}_{i j}, \quad j=1, \cdots, n$. A simple calculation using the error term yields the following error dynamics:

$$
\begin{aligned}
\dot{e}_{i 1}= & e_{i 2}-L a_{1} e_{i 1}+f_{i 1}(x, d(t)) \\
& \vdots \\
\dot{e}_{i(n-1)}= & e_{i n}-L^{n-1} a_{n-1} e_{i 1}+f_{i(n-1)}(x, d(t)) \\
\dot{e}_{i n}= & -L^{n} a_{n} e_{i 1}+f_{i n}(x, d(t)) .
\end{aligned}
$$

Next, we introduce the following change of coordinates $\xi_{i j}=\frac{e_{i j}}{L^{j-1}}, \quad j=1, \cdots, n$ to obtain a new error dynamic

$$
\dot{\xi}_{i}=L A \xi_{i}+\left[\begin{array}{l}
f_{i 1}(x, d(t)) \\
\frac{f_{i 2}(x, d(t))}{L} \\
\vdots \\
\frac{1}{L^{n-1}} f_{i n}(x, d(t))
\end{array}\right]
$$

where

$$
\xi_{i}=\left[\begin{array}{l}
\xi_{i 1} \\
\xi_{i 2} \\
\vdots \\
\xi_{i n}
\end{array}\right], \quad A=\left[\begin{array}{cccc}
-a_{1} & 1 & \cdots & 0 \\
\vdots & \vdots & \ddots & \vdots \\
-a_{n-1} & 0 & \cdots & 1 \\
-a_{n} & 0 & \cdots & 0
\end{array}\right]
$$

$A$ is a Hurwitz matrix. Therefore, there is a positive definite matrix $P=P^{T}>0$ such that

$$
A^{T} P+P A=-I
$$


Consider the Lyapunov function $V_{\xi_{i}}=\xi_{i}^{T} P \xi_{i}$. The derivative of $V_{\xi_{i}}$ along (3.2) is,

$$
\begin{aligned}
\dot{V}_{\xi_{i}} & =-L\left\|\xi_{i}\right\|^{2}+2 \xi_{i}^{T} P\left[\begin{array}{c}
f_{i 1}(x, d(t)) \\
\frac{f_{i 2}(x, d(t))}{L} \\
\vdots \\
\frac{f_{i n}(x, d(t))}{L^{n-1}}
\end{array}\right] \\
& \leq-L\left\|\xi_{i}\right\|^{2}+2\|P\|\left\|\xi_{i}\right\|\left(\left|f_{i 1}\right|+\frac{1}{L}\left|f_{i 2}\right|+\cdots+\frac{1}{L^{n-1}}\left|f_{i n}\right|\right) .
\end{aligned}
$$

By Assumption 2.1,

$$
\begin{aligned}
\left|f_{i 1}(x, d(t))\right| \leq & c\left(\left|x_{11}\right|+\cdots+\left|x_{m 1}\right|\right) \\
\frac{\left|f_{i 2}(x, d(t))\right|}{L} \leq & \frac{c}{L}\left(\left|x_{11}\right|+\cdots+\left|x_{m 1}\right|+\left|x_{12}\right|+\cdots+\left|x_{m 2}\right|\right) \\
& \vdots \\
\frac{\left|f_{i n}(x, d(t))\right|}{L^{n-1} \leq} & \frac{c}{L^{n-1}}\left(\left|x_{11}\right|+\cdots+\left|x_{m 1}\right|+\left|x_{12}\right|+\cdots\right. \\
& \left.+\left|x_{m 2}\right|+\cdots+\left|x_{1 n}\right|+\cdots+\left|x_{m n}\right|\right) .
\end{aligned}
$$

Therefore, letting $c_{1}=2\|P\| c$, we have

$$
\begin{aligned}
\dot{V}_{\xi_{i}} \leq & -L\left\|\xi_{i}\right\|^{2}+c_{1}\left\|\xi_{i}\right\|\left[\left(1+\frac{1}{L}+\cdots+\frac{1}{L^{n-1}}\right)\left|x_{11}\right|+\left(\frac{1}{L}+\cdots+\frac{1}{L^{n-1}}\right)\left|x_{12}\right|\right. \\
& +\cdots+\frac{1}{L^{n-1}}\left|x_{1 n}\right|+\left(1+\frac{1}{L}+\cdots+\frac{1}{L^{n-1}}\right)\left|x_{21}\right|+\left(\frac{1}{L}+\cdots+\frac{1}{L^{n-1}}\right)\left|x_{22}\right| \\
& +\cdots+\frac{1}{L^{n-1}}\left|x_{2 n}\right|+\cdots+\left(1+\frac{1}{L}+\cdots+\frac{1}{L^{n-1}}\right)\left|x_{m 1}\right| \\
& \left.+\left(\frac{1}{L}+\cdots+\frac{1}{L^{n-1}}\right)\left|x_{m 2}\right|+\cdots+\frac{1}{L^{n-1}}\left|x_{m n}\right|\right] \\
\leq & -L\left\|\xi_{i}\right\|^{2}+c_{1}\left\|\xi_{i}\right\|\left(\left[n\left|x_{11}\right|+\frac{1}{L^{n-1}}\left|x_{12}\right|+\cdots+\frac{1}{L^{n-1}}\left|x_{1 n}\right|+n\left|x_{21}\right|\right.\right. \\
& \left.\left.+\frac{n-1}{L}\left|x_{22}\right|+\cdots+\frac{1}{L^{n-1}}\left|x_{2 n}\right|+\cdots+n\left|x_{m 1}\right|+\cdots+\frac{1}{L^{n-1}}\left|x_{m n}\right|\right]\right) \\
\leq & -L\left\|\xi_{i}\right\|^{2}+c_{2}\left\|\xi_{i}\right\|\left[\left(\left|x_{11}\right|+\cdots+\left|x_{m 1}\right|\right)+\frac{1}{L}\left(\left|x_{12}\right|+\cdots+\left|x_{m 2}\right|\right)+\cdots\right. \\
& \left.+\frac{1}{L^{n-1}}\left(\left|x_{1 n}\right|+\cdots+\left|x_{m n}\right|\right)\right],
\end{aligned}
$$

for a constant $c_{2}>0$. Define $z_{i j}=\frac{\hat{x}_{i j}}{L^{j-1}}, \quad j=1, \cdots, n$. This, together with the fact that $x_{i j}=\hat{x}_{i j}+L^{j-1} \xi_{i j}$, implies

$$
\left|\frac{1}{L^{j-1}} x_{i j}\right| \leq\left|\frac{1}{L^{j-1}} \hat{x}_{i j}\right|+\left|\xi_{i j}\right|=\left|z_{i j}\right|+\left|\xi_{i j}\right|, \quad j=1, \cdots, n .
$$

With this in mind, it is not difficult to deduce that

$$
\begin{aligned}
\dot{V}_{\xi_{i}} \leq & -L\left\|\xi_{i}\right\|^{2}+\sqrt{n} c_{2}\left\|\xi_{i}\right\|\left(\left\|z_{1}\right\|+\cdots+\left\|z_{m}\right\|+\left\|\xi_{1}\right\|+\cdots+\left\|\xi_{m}\right\|\right) \\
\leq & -L\left\|\xi_{i}\right\|^{2}+c_{3}\left\|z_{1}\right\|^{2}+c_{3}\left\|z_{2}\right\|^{2}+\cdots \\
& +c_{3}\left\|z_{m}\right\|^{2}+c_{3}\left\|\xi_{1}\right\|^{2}+c_{3}\left\|\xi_{2}\right\|^{2}+\cdots+c_{3}\left\|\xi_{m}\right\|^{2}
\end{aligned}
$$


for a constant $c_{3}>0$.

\section{B. CONTROLLER DESIGN}

Under the new coordinates $z_{i j}=\frac{\hat{x}_{i j}}{L^{j-1}}, \quad j=1, \cdots, n$, system (3.1) becomes

$$
\begin{gathered}
\dot{z}_{i 1}=L z_{i 2}+L a_{1} \xi_{i 1} \\
\vdots \\
\dot{z}_{i(n-1)}=L z_{i n}+L a_{n-1} \xi_{i 1} \\
\dot{z}_{i n}=\frac{1}{L^{n-1}} u_{i}+L a_{n} \xi_{i 1} .
\end{gathered}
$$

Construct $u_{i}=-L^{n}\left[k_{1} z_{i 1}+k_{2} z_{i 2}+\cdots+k_{n} z_{i n}\right]$, where $k_{1}, \cdots, k_{n}$ are the coefficients of the Hurwitz polynomial $s^{n}+k_{n} s^{n-1}+\cdots+k_{2} s+k_{1}=0$. Under this controller, system (3.4) can be written in the following compact form,

$$
\dot{z}_{i}=L B z_{i}+L D \xi_{i 1}
$$

where

$$
z_{i}=\left[\begin{array}{l}
z_{i 1} \\
z_{i 2} \\
\vdots \\
z_{i n}
\end{array}\right], \quad D=\left[\begin{array}{l}
a_{1} \\
a_{2} \\
\vdots \\
a_{n}
\end{array}\right], \quad B=\left[\begin{array}{cccc}
0 & 1 & \cdots & 0 \\
\vdots & \vdots & \ddots & \vdots \\
0 & 0 & \cdots & 1 \\
-k_{1} & -k_{2} & \cdots & -k_{n}
\end{array}\right] .
$$

For Hurwitz matrix $B$, there is a positive definite matrix $Q=Q^{T}>0$ such that

$$
B^{T} Q+Q B=-I
$$

Consider the following Lyapunov function $V_{z_{i}}=z_{i}^{T} Q z_{i}$. By the necessary substitution we are arrive at the following equations,

$$
\begin{aligned}
\dot{V}_{z_{i}} & =-L\left\|z_{i}\right\|^{2}+2 z_{i}^{T} L Q D \xi_{i 1} \\
& \leq-L\left\|z_{i}\right\|^{2}+L c_{4}\left\|z_{i}\right\|\left\|\xi_{i}\right\|, \quad c_{4}=2\|Q D\|, \\
& \leq-\frac{1}{2} L\left\|z_{i}\right\|^{2}+L c_{5}\left\|\xi_{i}\right\|^{2}, \quad c_{5}=\frac{c_{4}^{2}}{2} .
\end{aligned}
$$

Construct the following Lyapunov function

$$
W_{i}=\left(1 / 2+c_{5}\right) V_{\xi_{i}}+V_{z_{i}} .
$$

Using equations (3.3) and (3.6), one has

$$
\begin{aligned}
\dot{W}_{i}= & \left(1 / 2+c_{5}\right) \dot{V}_{\xi_{i}}+\dot{V}_{z_{i}} \\
\leq & -L\left(1 / 2+c_{5}\right)\left\|\xi_{i}\right\|^{2}+c_{6}\left\|z_{1}\right\|^{2}+c_{6}\left\|z_{2}\right\|^{2}+\cdots+c_{6}\left\|z_{m}\right\|^{2} \\
& +c_{6}\left\|\xi_{1}\right\|^{2}+c_{6}\left\|\xi_{2}\right\|^{2}+\cdots+c_{6}\left\|\xi_{m}\right\|^{2}-\frac{1}{2} L\left\|z_{i}\right\|^{2}+L c_{5}\left\|\xi_{i}\right\|^{2} \\
= & -\frac{1}{2} L\left\|\xi_{i}\right\|^{2}-\frac{1}{2} L\left\|z_{i}\right\|^{2}+c_{6}\left\|z_{1}\right\|^{2}+\cdots+c_{6}\left\|z_{m}\right\|^{2} \\
& +c_{6}\left\|\xi_{1}\right\|^{2}+\cdots+c_{6}\left\|\xi_{m}\right\|^{2}
\end{aligned}
$$


where $c_{6}=\left(1 / 2+c_{5}\right) c_{3}$. Consequently, for $m$ subsystems we have

$$
\sum_{i=1}^{m} \dot{W}_{i} \leq-\left(\frac{1}{2} L-m c_{6}\right) \sum_{i=1}^{m}\left\|\xi_{i}\right\|^{2}-\left(\frac{1}{2} L-m c_{6}\right) \sum_{i=1}^{m}\left\|z_{i}\right\|^{2} .
$$

If the gain $\mathrm{L}$ is made large enough, the right hand side of (3.8) will be negative definite. Hence, the closed-loop system will be globally exponentially stable (GES).

REMARK 3.2. In contrast to the common observer design that typically uses a copy of the nonlinear system, we design only a linear observer for each subsystem in the large-scale system (2.1). Such a construction has enabled us to deal with difficult issues caused by the uncertainties or nonlinearities of the systems in the single system case [13]. In this paper, this new construction of the observer and controller also lets us avoid dealing with the nonlinear functions of the interconnected unmeasurable states. Consequently, this feedback domination design leads to a solution to the problem of decentralized output feedback control of system (2.1).

REMARK 3.3. It is worthwhile pointing out that the observer and controller for each system have the same structure. Hence, after we construct one output feedback controller for one of the subsystems, we can duplicate the controller for the other $m-1$ subsystems. This property will reduce the design time and implementation cost for the control design of system (2.1).

REMARK 3.4. Note that in system (2.1) all the subsystems have the same dimension (i.e. $n$ ). However, if the dimensions of $m$ subsystems are different, we are still able to achieve similar stabilization result under Assumption 2.1 with different dimensional variables. The only difference is that the dimension of the observer will be consistent with the dimension of the corresponding subsystem.

In what follows, a two-system model will be simulated based on the design procedures developed for output feedback domination with high gain.

EXAMPLE 3.5. Consider the following interconnected nonlinear system,

$$
\begin{array}{ll}
x_{1}=x_{2} & \dot{y}_{1}=y_{2} \\
\dot{x}_{2}=u+y_{2} \sin x_{2} & \dot{y}_{2}=v+d(t) \ln \left(1+y_{2}^{2}\right)+d(t) x_{2} \\
x_{\text {output }}=x_{1} & y_{\text {output }}=y_{1}
\end{array}
$$

where $d(t)$ is a disturbance bounded by a known constant. As (3.9) shows, the $x$ system and $y$-system are coupled through the unmeasurable states $\left(x_{2}, y_{2}\right)$. Moreover, the unmeasurable states are associated with unknown disturbances. Therefore, most of the existing output feedback control design procedures will fail to be applicable to (3.9). On the other hand, it is easy to verify that Assumption 2.1 holds for (3.9). By Theorem 3.1, we are able to design an output feedback controller for (3.9). 

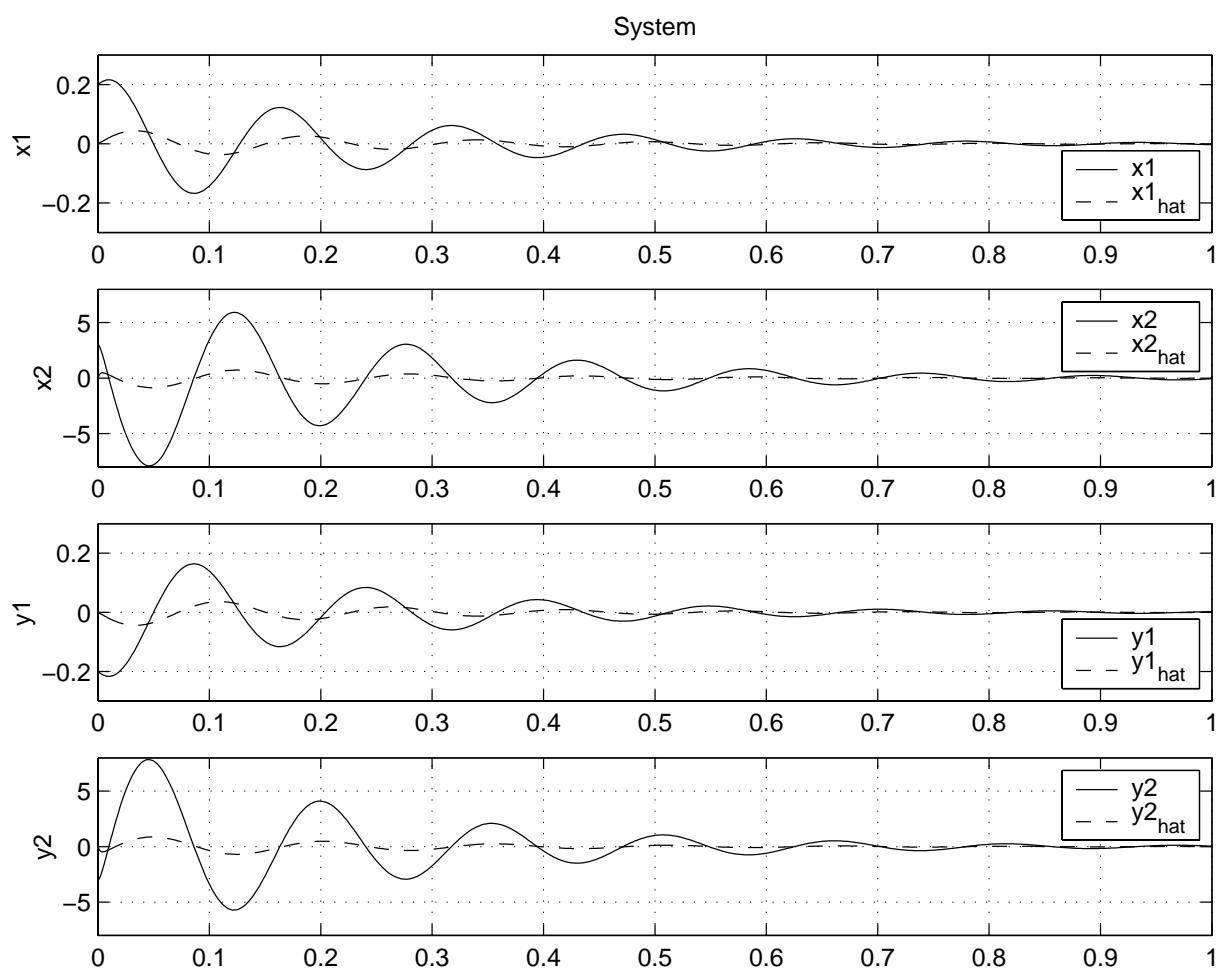

FIG. 1. $\left(x_{1}(0), x_{2}(0)\right)=(.2,3)\left(\hat{x}_{1}(0), \hat{x}_{2}(0)\right)=(0,0)\left(y_{1}(0), y_{2}(0)\right)=(-.2,-3)\left(\hat{y}_{1}(0), \hat{y}_{2}(0)\right)=$ $(0,0)$.

Specifically, we construct the observer as follows,

$$
\begin{aligned}
& \dot{\hat{x}}_{1}=\hat{x}_{2}+0.42 L\left(x_{1}-\hat{x}_{1}\right) \\
& \dot{\hat{x}}_{2}=u+4.2 L^{2}\left(x_{1}-\hat{x}_{1}\right) \\
& \dot{\hat{y}}_{1}=\hat{y}_{2}+0.42 L\left(y_{1}-\hat{y}_{1}\right) \\
& \dot{\hat{y}}_{2}=v+4.2 L^{2}\left(y_{1}-\hat{y}_{1}\right) .
\end{aligned}
$$

The control laws to be implemented are

$$
\begin{aligned}
& u=-28.6 L^{2} \hat{x}_{1}-25.7 L \hat{x}_{2} \\
& v=-28.6 L^{2} \hat{y}_{1}-25.7 L \hat{y}_{2},
\end{aligned}
$$

where the gain $\mathrm{L}$ was calculated to be 20. Figure 1 illustrates the response of the closed-loop system (3.9)-(3.10)-(3.11).

REMARK 3.6. As shown in [13], the output feedback domination design has the universal property that enables us to use a single output feedback controller to stabilize a family of nonlinear systems satisfying the same growth condition. This property is 


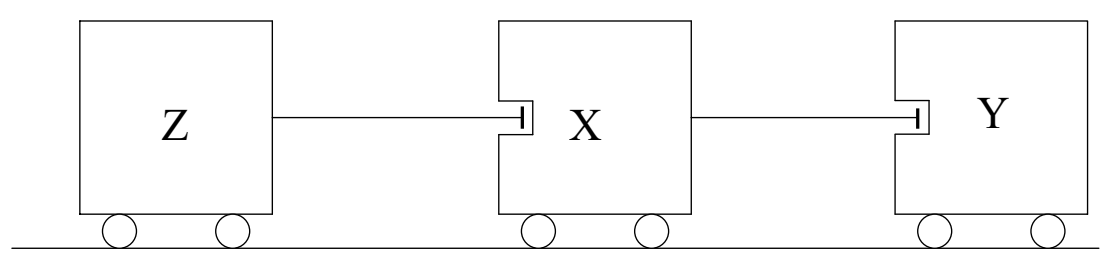

FIG. 2. Three Vehicles Linked by Dampers.

also valid in the decentralized case. For example, the same output feedback controller (3.10)-(3.11) for (3.9) will also stabilize the following system,

$$
\begin{array}{ll}
x_{1}=x_{2} & \dot{y}_{1}=y_{2} \\
\dot{x}_{2}=u+d(t) \sqrt{\left|x_{2} y_{2}\right|} & \dot{y}_{2}=v+d(t)\left(1-e^{-\left|y_{2}\right|}\right)+x_{2} \sin x_{2} \\
x_{\text {output }}=x_{1} & y_{\text {output }}=y_{1} .
\end{array}
$$

EXAmple 3.7 .

Figure 2 illustrates a practical application of the high gain technique for interconnected systems that was discussed in this section. Figure 2 has three vehicles that are interconnected by dampers and can be modeled by the following system of equations,

$$
\begin{array}{ll}
\dot{x}_{1}=x_{2} & \dot{y}_{1}=y_{2} \\
\dot{x}_{2}=u+f(t)\left(y_{2}-2 x_{2}+z_{2}\right) & \dot{y}_{2}=v+f(t)\left(x_{2}-y_{2}\right) \\
x_{\text {output }}=x_{1} & y_{\text {output }}=y_{1} \\
\dot{z}_{1}=z_{2} & \\
\dot{z}_{2}=w+f(t)\left(x_{2}-z_{2}\right) & \\
z_{\text {output }}=z_{1} &
\end{array}
$$

where $f(t) \in[-1,+1]$ is an uncertain damping function, and $x_{1}, y_{1}$, and $z_{1}$ are the positions for each vehicle respectively. A typical control objective for system (3.13) is as a reference or tracking problem where the connected vehicles are required to follow a particular trajectory. Closely examining system (3.13), it is apparent that the linear growth condition of Assumption 2.1 is met and therefore an output feedback controller can be designed based on Theorem 3.1. 
4. Output Feedback Domination with Low Gain. We now extend our results from the previous section to examine the global stabilization of upper-triangular systems. The decentralized control of a nonlinear upper-triangular system which has subsystems that are interconnected not only by their measured states but also by their unmeasurable states is a challenging problem due to the many practical applications that can be characterized as being in an upper-triangular form. The control of many mechanically underactuated systems can be modeled as an upper-triangular [16] system. One such decentralized control problem is the formation control of a group of carts with inverted pendulums. Another interconnected upper-triangular stabilization problem is the vertical landing of an autonomous aircraft onto the pitching deck of a ship. The issue of stabilization of upper-triangular nonlinear systems is of immense practical importance due to the many technical applications that exist in this area. Unfortunately, this area is not well studied and little research has been done in either state feedback or output feedback stabilization methods for upper-triangular nonlinear systems.

In this section we implement a linear output feedback controller to stabilize a nonlinear interconnected system under the following upper-triangular growth condition.

Assumption 4.1. For $i=1, \cdots, m$ and $j=1, \cdots, n-1$, there is a constant $c \geq 0$ such that

$$
\begin{aligned}
\left|f_{i j}(x, u(t))\right| \leq & c\left(\left|x_{1(j+2)}\right|+\cdots+\left|x_{1(n+1)}\right|+\left|x_{2(j+2)}\right|+\cdots+\left|x_{2(n+1)}\right|+\cdots\right. \\
& \left.+\left|x_{m(j+2)}\right|+\cdots+\left|x_{m(n+1)}\right|\right) \\
\left|f_{i n}(x, u(t))\right|= & 0, \quad \forall \quad x, u, t
\end{aligned}
$$

where $x_{i(n+1)}=u_{i}$.

THEOREM 4.2. Under Assumption 4.1, there exists a linear output feedback controller (2.3) that renders an upper-triangular interconnected system of the form of system (2.1) globally exponentially stable.

Proof. In order to prove Theorem 4.2, we follow a proof similar in structure to the previous section on lower-triangular systems but with the difference being that a small gain needs to be carefully chosen in order to prove that the closed loop system is globally exponentially stable. 


\section{A. LINEAR OBSERVER DESIGN}

We begin by designing the following linear observer for subsystem $i$

$$
\begin{aligned}
\dot{\hat{x}}_{i 1}= & \hat{x}_{i 2}+\varepsilon a_{1}\left(x_{i 1}-\hat{x}_{i 1}\right) \\
\dot{\hat{x}}_{i 2}= & \hat{x}_{i 3}+\varepsilon^{2} a_{2}\left(x_{i 1}-\hat{x}_{i 1}\right) \\
& \vdots \\
\dot{\hat{x}}_{i(n-1)}= & \hat{x}_{i n}+\varepsilon^{(n-1)} a_{n-1}\left(x_{i 1}-\hat{x}_{i 1}\right) \\
\dot{\hat{x}}_{i n}= & u_{i}+\varepsilon^{n} a_{n}\left(x_{i 1}-\hat{x}_{i 1}\right)
\end{aligned}
$$

where $0<\varepsilon<1$ is a gain parameter to be determined later, and $a_{j}>0, j=1, \cdots, n$, are coefficients of the Hurwitz polynomial $p(s)=s^{n}+a_{1} s^{(n-1)}+\cdots+a_{n-1} s+a_{n}$. Similar to the previous section, by defining the error term $e_{i j}=x_{i j}-\hat{x}_{i j}, \quad j=$ $1, \cdots, n$, and the change of coordinates $\xi_{i j}=\frac{e_{i j}}{\varepsilon^{j-1}}, \quad j=1, \cdots, n$, we have the following error dynamics

$$
\dot{\xi}_{i}=\varepsilon A \xi_{i}+\left[\begin{array}{c}
f_{i 1}(x, u(t), d(t)) \\
\frac{f_{i 2}(x, u(t), d(t))}{\varepsilon} \\
\vdots \\
\frac{f_{i n-2}(x, u(t), d(t))}{\varepsilon^{n-3}(t)} \\
\frac{f_{i(n-1)}(x, u(t), d(t))}{\varepsilon^{n-2}} \\
0
\end{array}\right]
$$

with

$$
\xi_{i}=\left[\begin{array}{l}
\xi_{i 1} \\
\xi_{i 2} \\
\vdots \\
\xi_{i n}
\end{array}\right], \quad A=\left[\begin{array}{cccc}
-a_{1} & 1 & \cdots & 0 \\
\vdots & \vdots & \ddots & \vdots \\
-a_{n-1} & 0 & \cdots & 1 \\
-a_{n} & 0 & \cdots & 0
\end{array}\right]
$$

Consider the Lyapunov function $V_{\xi_{i}}=\xi_{i}^{T} P \xi_{i}$, where $P$ is a positive definite matrix $P=P^{T}>0$ such that

$$
A^{T} P+P A=-I
$$

The derivative of $V_{\xi_{i}}$ along (4.2) is,

$$
\begin{aligned}
\dot{V}_{\xi_{i}} & =\varepsilon \xi_{i}^{T}\left(A^{T} P+P A\right) \xi_{i}+2 \xi_{i}^{T} P\left[f_{i 1}(x, d(t)), \ldots, \frac{f_{i(n-1)}(x, u(t), d(t))}{\varepsilon^{n-2}}, 0\right]^{T} \\
& \leq-\varepsilon\left\|\xi_{i}\right\|^{2}+c_{0}\left\|\xi_{i}\right\|\left(\left|f_{i 1}\right|+\frac{1}{\varepsilon}\left|f_{i 2}\right|+\cdots+\frac{1}{\varepsilon^{n-2}}\left|f_{i(n-1)}\right|+0\right) \\
c_{0} & =2\|P\| .
\end{aligned}
$$


Based on Assumption4.1,

$$
\begin{aligned}
\left|f_{i 1}(x, d(t))\right| \leq & c\left(\left|x_{13}\right|+\cdots+\left|x_{1(n+1)}\right|+\cdots+\left|x_{i 3}\right|+\cdots\right. \\
& \left.+\left|x_{i(n+1)}\right|+\cdots+\left|x_{m 3}\right|+\cdots+\left|x_{m(n+1)}\right|\right) \\
\frac{\left|f_{i 2}(x, d(t))\right|}{\varepsilon} \leq & \frac{1}{\varepsilon} c\left(\left|x_{14}\right|+\cdots+\left|x_{1(n+1)}\right|+\cdots+\left|x_{i 4}\right|+\cdots\right. \\
& \left.+\left|x_{i(n+1)}\right|+\cdots+\left|x_{m 4}\right|+\cdots+\left|x_{m(n+1)}\right|\right) \\
\vdots & \\
\frac{\left|f_{i(n-2)}(x, d(t))\right|}{\varepsilon^{n-3} \leq} & \frac{1}{\varepsilon^{n-3}} c\left(\left|x_{1 n}\right|+\left|x_{1(n+1)}\right|+\cdots+\left|x_{i n}\right|\right. \\
& \left.+\left|x_{i(n+1)}\right|+\cdots+\left|x_{m n}\right|+\left|x_{m(n+1)}\right|\right) \\
\frac{\left|f_{i(n-1)}(x, d(t))\right|}{\varepsilon^{n-2}} \leq & \frac{1}{\varepsilon^{n-2}} c\left(\left|u_{1}\right|+\cdots+\left|u_{m}\right|\right) .
\end{aligned}
$$

Therefore,

$$
\begin{aligned}
& c_{0}\left(\left|f_{i 1}\right|+\frac{1}{\varepsilon}\left|f_{i 2}\right|+\cdots+\frac{1}{\varepsilon^{n-2}}\left|f_{i(n-1)}\right|\right) \\
\leq & c_{1}\left(\left|x_{13}\right|+\left(1+\frac{1}{\varepsilon}\right)\left|x_{14}\right|+\left(1+\frac{1}{\varepsilon}+\frac{1}{\varepsilon^{2}}\right)\left|x_{15}\right|+\cdots\right. \\
& +\left(1+\frac{1}{\varepsilon}+\frac{1}{\varepsilon^{2}}+\cdots+\frac{1}{\varepsilon^{n-3}}\right)\left|x_{1 n}\right|+\left(1+\frac{1}{\varepsilon}+\frac{1}{\varepsilon^{2}}+\cdots+\frac{1}{\varepsilon^{n-2}}\right)\left|u_{1}\right| \\
& +\cdots+\left|x_{m 3}\right|+\left(1+\frac{1}{\varepsilon}\right)\left|x_{m 4}\right|+\left(1+\frac{1}{\varepsilon}+\frac{1}{\varepsilon^{2}}\right)\left|x_{m 5}\right|+\cdots \\
& \left.+\left(1+\frac{1}{\varepsilon}+\frac{1}{\varepsilon^{2}}+\cdots+\frac{1}{\varepsilon^{n-3}}\right)\left|x_{m n}\right|+\left(1+\frac{1}{\varepsilon}+\frac{1}{\varepsilon^{2}}+\cdots+\frac{1}{\varepsilon^{n-2}}\right)\left|u_{m}\right|\right)
\end{aligned}
$$

where $c_{1}=c \times c_{0}$. Assuming that $\varepsilon$ is a small gain, we have the following condition,

$$
\left(1+\frac{1}{\varepsilon}+\cdots+\frac{1}{\varepsilon^{i-1}}\right) \leq \frac{1}{\varepsilon^{i-1}}(i) \leq \frac{1}{\varepsilon^{i-1}}(n-1),
$$

where $i \leq(n-1)$. Therefore we can develop the following equation,

$$
\begin{aligned}
& c_{0}\left(\left|f_{i 1}\right|+\frac{1}{\varepsilon}\left|f_{i 2}\right|+\cdots+\frac{1}{\varepsilon^{n-2}}\left|f_{i(n-1)}\right|\right) \\
\leq & c_{2}\left(\left|x_{13}\right|+\frac{1}{\varepsilon}\left|x_{14}\right|+\frac{1}{\varepsilon^{2}}\left|x_{15}\right|+\cdots+\frac{1}{\varepsilon^{n-3}}\left|x_{1 n}\right|+\frac{1}{\varepsilon^{n-2}}\left|u_{1}\right|\right. \\
& \left.+\cdots+\left|x_{m 3}\right|+\frac{1}{\varepsilon}\left|x_{m 4}\right|+\frac{1}{\varepsilon^{2}}\left|x_{m 5}\right|+\cdots+\frac{1}{\varepsilon^{n-3}}\left|x_{m n}\right|+\frac{1}{\varepsilon^{n-2}}\left|u_{m}\right|\right),
\end{aligned}
$$

for a constant $c_{2}=(n-1) c_{1}$. With this in mind, Equation (4.4) becomes,

$$
\begin{aligned}
& \dot{V}_{\xi_{i}} \leq-\varepsilon\left\|\xi_{i}\right\|^{2}+c_{2}\left\|\xi_{i}\right\|\left[\left(\left|x_{13}\right|+\left|x_{23}\right|+\cdots+\left|x_{m 3}\right|\right)+\frac{1}{\varepsilon}\left(\left|x_{14}\right|+\left|x_{24}\right|+\cdots+\left|x_{m 4}\right|\right)\right. \\
&+\frac{1}{\varepsilon^{2}}\left(\left|x_{15}\right|+\left|x_{25}\right|+\cdots+\left|x_{m 5}\right|\right)+\cdots+\frac{1}{\varepsilon^{n-3}}\left(\left|x_{1 n}\right|+\left|x_{2 n}\right|+\cdots+\left|x_{m n}\right|\right) \\
&\left.(4.4) \quad+\frac{1}{\varepsilon^{n-2}}\left(\left|u_{1}\right|+\cdots+\left|u_{m}\right|\right)\right] .
\end{aligned}
$$


Define $z_{i j}=\frac{\hat{x}_{i j}}{\varepsilon^{j-1}}, \quad j=1, \cdots, n$. This, together with the fact that $x_{i j}=\hat{x}_{i j}+\varepsilon^{j-1} \xi_{i j}$, implies

$$
\left|\frac{1}{\varepsilon^{j-1}} x_{i j}\right| \leq\left|z_{i j}\right|+\left|\xi_{i j}\right|, \quad j=1, \cdots, n .
$$

\section{B. CONTROLLER DESIGN}

We now construct the controller as follows,

$$
\begin{aligned}
u_{i} & =-\varepsilon^{n} k_{1} \hat{x}_{i 1}-\varepsilon^{n-1} k_{2} \hat{x}_{i 2}-\cdots-\varepsilon^{2} k_{n-1} \hat{x}_{i(n-1)}-\varepsilon k_{n} \hat{x}_{i n} \\
& =\varepsilon^{n}\left[-k_{1},-k_{2}, \cdots,-k_{n}\right]\left[\begin{array}{c}
z_{i 1} \\
z_{i 2} \\
\vdots \\
z_{i n}
\end{array}\right]
\end{aligned}
$$

where $k_{1}, \cdots, k_{n}$ are the coefficients of the Hurwitz polynomial $s^{n}+k_{n} s^{n-1}+\cdots+$ $k_{2} s+k_{1}=0$. Clearly $\frac{\left|u_{i}\right|}{\varepsilon^{n-2}} \leq \varepsilon^{2} \bar{c}_{2}\left\|z_{i}\right\|$, for a constant $\bar{c}_{2}>0$.

Using the relationship from Equation (4.4),

$$
\begin{aligned}
\dot{V}_{\xi_{i}} \leq & -\varepsilon\left\|\xi_{i}\right\|^{2}+c_{2}\left\|\xi_{i}\right\| \varepsilon^{2}\left[\left|z_{13}\right|+\left|z_{23}\right|+\cdots+\left|z_{m 3}\right|\right. \\
& \left.+\left|z_{14}\right|+\left|z_{24}\right|+\cdots+\left|z_{m 4}\right|+\cdots+\left|z_{1 n}\right|+\left|z_{2 n}\right|+\cdots+\left|z_{m n}\right|\right] \\
& +\left|\xi_{13}\right|+\left|\xi_{23}\right|+\cdots+\left|\xi_{m 3}\right|+\left|\xi_{14}\right|+\left|\xi_{24}\right|+\cdots+\left|\xi_{m 4}\right| \\
& \left.+\cdots+\left|\xi_{m n}\right|+\bar{c}_{2}\left(\left\|z_{1}\right\|+\cdots+\left\|z_{m}\right\|\right)\right] \\
\leq & -\varepsilon\left\|\xi_{i}\right\|^{2}+c_{3} \varepsilon^{2}\left\|z_{1}\right\|^{2}+c_{3} \varepsilon^{2}\left\|z_{2}\right\|^{2}+\cdots \\
& +c_{3} \varepsilon^{2}\left\|z_{m}\right\|^{2}+c_{3} \varepsilon^{2}\left\|\xi_{1}\right\|^{2}+c_{3} \varepsilon^{2}\left\|\xi_{2}\right\|^{2}+\cdots+c_{3} \varepsilon^{2}\left\|\xi_{m}\right\|^{2},
\end{aligned}
$$

for a constant $c_{3}>0$. Using the new coordinates $z_{i j}=\frac{\hat{x}_{i j}}{\varepsilon^{j-1}}$ and the controller (4.5), system (4.1) can be written in the following compact form,

$$
\dot{z}_{i}=\varepsilon B z_{i}+\varepsilon D \xi_{i 1}
$$

with

$$
z_{i}=\left[\begin{array}{l}
z_{i 1} \\
z_{i 2} \\
\vdots \\
z_{i n}
\end{array}\right], \quad D=\left[\begin{array}{l}
a_{1} \\
a_{2} \\
\vdots \\
a_{n}
\end{array}\right], \quad B=\left[\begin{array}{cccc}
0 & 1 & \cdots & 0 \\
\vdots & \vdots & \ddots & \vdots \\
0 & 0 & \cdots & 1 \\
-k_{1} & -k_{2} & \cdots & -k_{n}
\end{array}\right]
$$

Consider the following Lyapunov function $V_{z_{i}}=z_{i}^{T} Q z_{i}$ where $P$ is a positive definite matrix $Q=Q^{T}>0$ such that

$$
B^{T} Q+Q B=-I
$$


By the necessary substitution we arrive at the following equations,

$$
\begin{aligned}
\dot{V}_{z_{i}} & \leq-\varepsilon\left\|z_{i}\right\|^{2}+\varepsilon 2 z_{i}^{T} Q D \xi_{i 1} \\
& \leq-\varepsilon\left\|z_{i}\right\|^{2}+\varepsilon c_{4}\left\|z_{i}\right\|\left\|\xi_{i}\right\|, \quad c_{4}=2\|Q D\| .
\end{aligned}
$$

By applying the following inequality,

$$
\left\|z_{i}\right\| c_{4}\left\|\xi_{i}\right\| \leq \frac{\left\|z_{i}\right\|^{2}}{2}+\frac{c_{4}^{2}}{2}\left\|\xi_{i}\right\|^{2}
$$

the following equation is constructed,

$$
\dot{V}_{z_{i}} \leq-\frac{1}{2} \varepsilon\left\|z_{i}\right\|^{2}+\varepsilon c_{5}\left\|\xi_{i}\right\|^{2}, \quad c_{5}=\frac{c_{4}^{2}}{2}
$$

Construct the following Lyapunov function

$$
W_{i}=\left(1 / 2+c_{5}\right) V_{\varepsilon_{i}}+V_{z_{i}} .
$$

Using equations (4.6) and (4.8), the following Lyapunov function is developed,

$$
\begin{aligned}
\dot{W}_{i}= & \left(1 / 2+c_{5}\right) \dot{V}_{\xi_{i}}+\dot{V}_{z_{i}} \\
\leq & -\varepsilon\left(1 / 2+c_{5}\right)\left\|\xi_{i}\right\|^{2}+c_{6} \varepsilon^{2}\left\|z_{1}\right\|^{2}+c_{6} \varepsilon^{2}\left\|z_{2}\right\|^{2} \\
& +\cdots+c_{6} \varepsilon^{2}\left\|z_{m}\right\|^{2}+c_{6} \varepsilon^{2}\left\|\xi_{1}\right\|^{2}+c_{6} \varepsilon^{2}\left\|\xi_{2}\right\|^{2} \\
& +\cdots+c_{6} \varepsilon^{2}\left\|\xi_{m}\right\|^{2}-\frac{1}{2} \varepsilon\left\|z_{i}\right\|^{2}+\varepsilon c_{5}\left\|\xi_{i}\right\|^{2} \\
= & -\frac{1}{2} \varepsilon\left\|\xi_{i}\right\|^{2}-\frac{1}{2} \varepsilon\left\|z_{i}\right\|^{2}+c_{6} \varepsilon^{2}\left\|z_{1}\right\|^{2} \\
& +\cdots+c_{6} \varepsilon^{2}\left\|z_{m}\right\|^{2}+c_{6} \varepsilon^{2}\left\|\xi_{1}\right\|^{2}+\cdots+c_{6} \varepsilon^{2}\left\|\xi_{m}\right\|^{2}, \\
& c_{6}=\left(1 / 2+c_{5}\right) c_{3} .
\end{aligned}
$$

Consequently, for $m$ subsystems we have

$$
\begin{aligned}
\sum_{i=1}^{m} \dot{W}_{i} \leq & -\frac{1}{2} \varepsilon \sum_{i=1}^{m}\left\|\xi_{i}\right\|^{2}-\frac{1}{2} \varepsilon \sum_{i=1}^{m}\left\|z_{i}\right\|^{2}+c_{6} m\left\|z_{1}\right\|^{2} \\
& +\cdots+c_{6} m\left\|z_{m}\right\|^{2}+c_{6} m\left\|\xi_{1}\right\|^{2}+\cdots+c_{6} m\left\|\xi_{m}\right\|^{2} \\
= & -\left(\frac{1}{2} \varepsilon-m c_{6} \varepsilon^{2}\right) \sum_{i=1}^{m}\left\|\xi_{i}\right\|^{2}-\left(\frac{1}{2} \varepsilon-m c_{6} \varepsilon^{2}\right) \sum_{i=1}^{m}\left\|z_{i}\right\|^{2}
\end{aligned}
$$

If the gain $\varepsilon$ is made small enough, the right hand side of Equation (4.10) will be negative definite. Hence, the closed-loop system will be GES.

REMARK 4.3. In the preceding section, the gain $L$ that was calculated had to be chosen in such a way that if large enough, the lower-triangular system was GES. Conversely, when dealing with the output feedback stabilization problem for an uppertriangle system in the form of system (2.1), the gain $\varepsilon$ must be made small enough to guarantee GES. Furthermore, the gain $\varepsilon$ provides a lower bound for stability and is therefore a quite conservative value. 
We conclude this section with the following example.

EXAMPLE 4.4. Consider the following nonlinear upper-triangular interconnected system,

$$
\begin{array}{ll}
\dot{x}_{1}=x_{2}+x_{3} \sin \left(y_{3}\right) d(t)+u^{\frac{1}{3}} v^{\frac{2}{3}} & \dot{y}_{1}=y_{2}+\ln \left(1+x_{3}^{2}\right) d(t) \\
\dot{x}_{2}=x_{3} & \dot{y}_{2}=y_{3} \\
\dot{x}_{3}=u & \dot{y}_{3}=v \\
x_{\text {output }}=x_{1} & y_{\text {output }}=y_{1}
\end{array}
$$

where $d(t)$ is a disturbance bounded by a known constant. As shown in system (4.11), the $x$-system and $y$-system are coupled or interconnected through the unmeasurable states $\left(x_{3}, y_{3}\right)$. Moreover, the unmeasurable states are associated with unknown disturbances. Therefore, most of the existing output feedback control design procedures will fail to be applicable to system (4.11). On the other hand, it is easy to verify that Assumption 4.1 holds for system (4.11). By Theorem 4.2, we are able to design an output feedback controller for system (4.11).

5. Extensions. In this section we will extend our results to deal with nontriangular systems that have a linear growth condition. Furthermore, we will examine the case when the growth rate $c$ is unknown for a system and show that by implementing a time-varying gain our previous results can still be employed.

5.1. Non-triangular Systems. We have proved the output feedback stabilization of large-scale systems with both lower-triangular and upper-triangular bounding functions. In the case when the system does not have a triangular bounding function, we can still develop a design procedure similar to the previous sections when the non-triangular system satisfies the following assumption.

Assumption 5.1. For $i=1, \cdots, m$ and $j=1, \cdots, n$, there is a constant $c \geq 0$ such that

$$
\begin{aligned}
\frac{\left|f_{i j}(x, d(t))\right|}{L^{j-1}} \leq & c\left[\left|x_{11}\right|+\cdots+\left|x_{m 1}\right|+\frac{1}{L}\left(\left|x_{2}\right|+\cdots+\left|x_{m 2}\right|\right)\right. \\
& \left.+\cdots+\frac{1}{L^{n-1}}\left(\left|x_{1 n}\right|+\cdots+\left|x_{m n}\right|\right)\right] .
\end{aligned}
$$

THEOREM 5.2. Under Assumption 5.1, there exists a linear output feedback controller (2.3) that renders the large-scale interconnected system (2.1) globally exponentially stable.

Proof. The proof is parallel to Theorem 3.1 and is omitted here for brevity.

Example 5.3. It can be shown that by applying Assumption 5.1, we can develop a controller (2.3) to such non-triangular systems as,

$$
\begin{array}{ll}
\dot{x}_{1}=x_{2} & \dot{y}_{1}=y_{2} \\
\dot{x}_{2}=x_{3}+\left|x_{3}\right|^{\frac{1}{2}} \ln \left(1+x_{1}^{2}\right) & \dot{y}_{2}=y_{3} \\
\dot{x}_{3}=u & \dot{y}_{3}=v \\
x_{\text {output }}=x_{1} & y_{\text {output }}=y_{1} .
\end{array}
$$


In system (5.2), the function $\dot{x}_{2}=x_{3}+\left|x_{3}\right|^{\frac{1}{2}} \ln \left(1+x_{1}^{2}\right)$ is not bounded by a lower -triangular function, therefore, Theorem 3.1 is not applicable. However recognizing that

$$
\frac{\left|x_{3}\right|^{\frac{1}{2}} \ln \left(1+x_{1}^{2}\right)}{L} \leq \ln ^{2}\left(1+x_{1}^{2}\right)+\frac{\left|x_{3}\right|}{L^{2}} \leq\left|x_{1}\right|+\frac{\left|x_{3}\right|}{L^{2}},
$$

a linear growth condition exists for Assumption 5.1. As a consequence, we can construct a controller by Theorem 5.2 such that the closed-loop system is globally exponentially stable.

5.2. Large-scale Systems with Unknown Growth Rate. For the interconnected system (2.1), there might be circumstances when the growth rate $c$ in Assumption 2.1 is unknown. In this section, we show that using the time-varying observer developed in [14], a decentralized output feedback controller with time-varying gain $L(t)$ can be designed to globally regulate system (2.1) whose nonlinear function $f_{i, j}(x, d(t))$ linearly grows at an unknown rate.

TheOrem 5.4. Suppose system (2.1) satisfies Assumption 2.1 with unknown growth rate $c$. Then, there exists an output feedback controller of the form,

$$
\begin{aligned}
& \dot{\xi}_{i}=M(t) \xi_{i}+N(t) y_{i}, \quad M(t) \in \mathbb{R}^{n \times n}, \quad N(t) \in \mathbb{R}^{n} \\
& u_{i}=K(t) \xi_{i}, \quad K(t) \in \mathbb{R}^{1 \times n}, i=1, \cdots, m,
\end{aligned}
$$

such that all the states of (2.1) and (5.3) are ultimately bounded. Moreover,

$$
\lim _{t \rightarrow+\infty}(x(t), \xi(t))=0 .
$$

Proof. Theorem 5.4 can be easily proved by combining the time-varying observer and controller proposed in [14] with the design procedure for Theorem 3.1. The linear structure of the observer will avoid the difficulties in dealing with the uncertain nonlinear functions while the time-varying gain will suppress the effects of the unknown growth rate. The proof is parallel to Theorem 3.1 and is omitted here for brevity.

EXAMPLE 5.5. We apply our time-varying result to system (4.11) to globally regulate by output feedback when $d(t)$ is bounded by an unknown constant $c$. By Theorem 5.4, the following observer is developed using the varying gain $L(t)$,

$$
\begin{aligned}
& \dot{\hat{x}}_{1}=\hat{x}_{2}+L(t)\left(x_{1}-\hat{x}_{1}\right) \\
& \dot{\hat{x}}_{2}=u+L^{2}(t)\left(x_{1}-\hat{x}_{1}\right) \\
& \dot{\hat{y}}_{1}=\hat{y}_{2}+L(t)\left(y_{1}-\hat{y}_{1}\right) \\
& \dot{\hat{y}}_{2}=v+L^{2}(t)\left(y_{1}-\hat{y}_{1}\right) .
\end{aligned}
$$

The control laws to be implemented are found to be

$$
\begin{aligned}
& u=-L^{2}(t) \hat{x}_{1}-L(t) \hat{x}_{2} \\
& v=-L^{2}(t) \hat{y}_{1}-L(t) \hat{y}_{2} .
\end{aligned}
$$



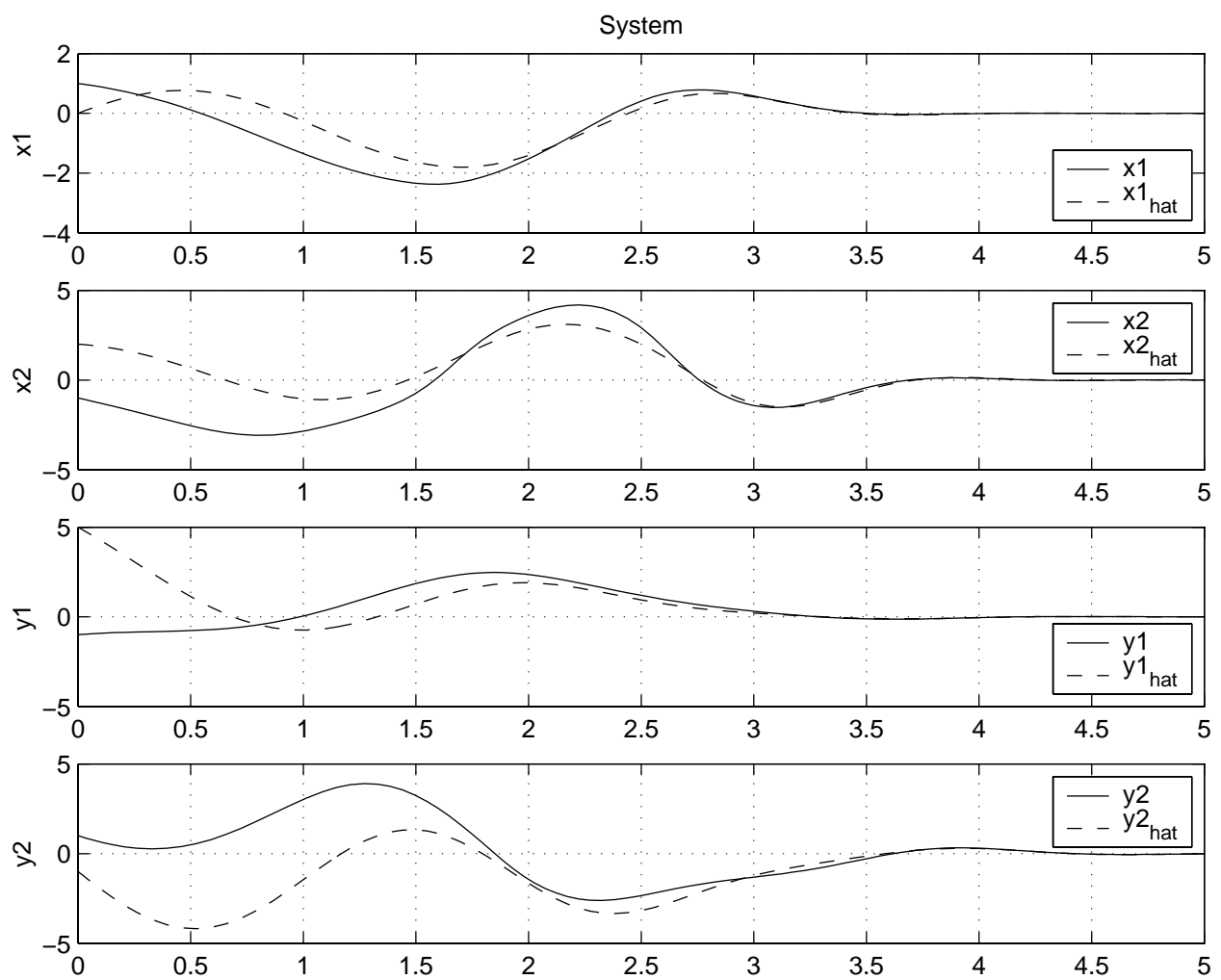

FIG. 3. $\left(x_{1}(0), x_{2}(0)\right)=(1,-1)\left(\hat{x}_{1}(0), \hat{x}_{2}(0)\right)=(0,2)\left(y_{1}(0), y_{2}(0)\right)=(-1,0)\left(\hat{y}_{1}(0), \hat{y}_{2}(0)\right)=$ $(5,-1)$.

Figure 3 illustrates the result due to an implementation of the varying gain $L(t)=t+1$.

REMARK 5.6. Using a similar argument proposed in [14], we can prove that $(x, y, \hat{x}, \hat{y})$ tend to zero exponentially. As a consequence, the observers and controllers are ultimately bounded even though $L(t)$ is not bounded. In fact, as shown in Figure 3 the states of the system (4.11) and observers (5.4) tend to zero very quickly. In an actual application of using an increasing $L(t)$ gain, to avoid the issue of an unbounded $L(t)$ we can saturate the gain after a sufficient amount of time has passed. To employ a saturation technique, a practical method would be to measure the error between the reference set point and the current output and after the error has become and remains acceptably small, the current value of the gain $L(t)$ can be made to no longer increase.

6. Conclusion. We have presented in this paper, a method of using output feedback to globally stabilize large-scale nonlinear systems whose $m$ subsystems are highly interconnected by unmeasurable states. Under the linear growth condition, we 
explicitly construct $m$ sets of linear observers and controllers by only using the output feedback information of each subsystem. It is shown that global output feedback stabilization is achieved for the closed-loop system. This design method was applied to lower-triangular, upper-triangular, and non-triangular nonlinear systems. Also, observers and controllers using time-varying gain are developed to control large-scale systems with unknown growth rates. The universal feature of our feedback domination

design enables us to design one output feedback controller and apply it to any system which satisfies the same growth condition.

\section{REFERENCES}

[1] W. Bachmann and D. Konik, On Stabilization of Interconnected Systems using Output Feedback, System Control Letter, 5(1984), pp. 89-95.

[2] E. J. DAvison, The Decentralized Stablization and Control of a Class of Unknown Nonlinear Time-Varying Systems, Automatica, 10:3(1974), pp. 309-316.

[3] P. Ionnnou, Decentralized Adaptive Control of Interconnected Systems, IEEE Trans. Aut. Contr., 31(1986), pp. 291-298.

[4] S. Jain and F. Khorrami, Decentralized Adaptive Output-Feedback Design for Large-Scale Nonlinear Systems, IEEE Trans. Automat. Contr., 42(2001), pp. 729-735.

[5] Z. P. JiAng, Decentralized and Adaptive Nonlinear Tracking of Large-Scale Systems via Output Feedback, IEEE Trans. Automat. Contr., 45(2000), pp. 2122-2128.

[6] Z. P. Jiang, F. Khorrami, And D. Hill, Decentralized Nonlinear Output-Feedback Stabilization with Disturbance Attenuation, IEEE Trans. Automat. Contr., 46(2001), pp. 16231629.

[7] H. Khalil And A SABeri, Decentralized stabilization of nonlinear interconnected systems using high-gain feedback, IEEE Trans. Automat. Contr., 27(1982), pp. 265-268.

[8] A. J. KRener And A. IsIDORI, Linearization by output injection and nonlinear observer, Systems \& Control Letters, 3(1983), pp. 47-52.

[9] A. J. Krener and W. Respondek, Nonlinear observers with linearizable error dynamics, SIAM J. Contr. Optimiz. 23(1985), pp. 197-216.

[10] B. Labibi, B. Lohmann, A. K. Sedigh, and P. J. Maralani, Output Feedback Decentralized Control of Large-Scale Systems Using Weighted Sensitivity Functions Minimization, Systems \& Control Letters, 47(2002), pp. 191-198.

[11] A. Linnemann, Decentralized Control of Dynamically Interconnected Systems, IEEE Trans. Aut. Contr., 29(1984), pp. 1052-1054.

[12] L. Praly, Asymptotic stabilization via output feedback for lower triangular systems with output dependent incremental rate, IEEE Trans. Aut. Contr., 48(2003), pp. 1103-1108.

[13] C. Qian And W. Lin, Output Feedback Control of a Class of Nonlinear Systems: A Nonseparation Principle Paradigm, IEEE Trans. Aut. Contr., 47(2002), pp. 1710-1715.

[14] C. Qian, C. B. Schrader, And W. Lin, Global Regulation of a Class of Uncertain Nonlinear Systems using Output Feedback, Proceedings of 2003 American Control Conference, Denver, CO, pp. 1542-1547, 2003.

[15] A. Saberi and H. K. Khalil, Decentralized Stabilization of Interconnected Systems using Output Feedback, Int. J. Contr., 41(1985), pp. 1461-1475.

[16] M. W. Spong, Underactuated Mechanical Systems, Control Problems in Robotics and Automation, B. Siciliano and K. P. Valavanis, editors, Springer-Verlag, London, UK, 1997.

[17] X. H. XIA AND W. B. GaO, Nonlinear observer design by observer error linearization, SIAM J. Contr. Optimiz. 27(1989), pp. 199-216. 
[18] D. Z. Zheng, Decentralized output feedback stabilization of a class of nonlinear interconnected systems, IEEE Trans. Aut. Contr., 34(1989), pp. 1297-1300. 